Research Article

\title{
Electrostrictive Mechanism of Radiation Self-Action in Nanofluids
}

\author{
Albert Livashvili, ${ }^{1}$ Victor Krishtop, ${ }^{2}$ and Margarita Yakunina ${ }^{1}$ \\ ${ }^{1}$ Mathematics Department, Far Eastern State Transport University, 47 Seryshev Street, Khabarovsk 680021, Russia \\ ${ }^{2}$ Physics Department, Far Eastern State Transport University, 47 Seryshev Street, Khabarovsk 680021, Russia
}

Correspondence should be addressed to Victor Krishtop; krishtop@list.ru

Received 7 June 2013; Accepted 24 August 2013

Academic Editor: Rosa Lukaszew

Copyright ( 2013 Albert Livashvili et al. This is an open access article distributed under the Creative Commons Attribution License, which permits unrestricted use, distribution, and reproduction in any medium, provided the original work is properly cited.

The electrostriction mechanism of beam self-focusing in nanofluids is theoretically investigated. An analytical solution of the diffusion equation, which describes the dynamics of particles in nanofluids, was obtained and studied. Explicit expressions for the nonlinear part of the refractive index and concentration lens focal length are presented. It is shown that there is a limit on the radiation intensity associated with the physical and hydrodynamic characteristics of the phenomena in these processes.

\section{Introduction}

Nanoparticles are being increasingly adopted in new and different areas of science and technology [1-4]. Colloidal suspensions that contain nanoparticles, also known as nanofluids, have found a variety of important applications in modern technologies [5]. For example, magnetic fluids are largely used for polishing optical components $[6,7]$, suspensions of silica particles in liquid crystals have exhibited extraordinary capabilities for optical storage $[8,9]$, and artificial media with a high optical nonlinearity were obtained in colloids of submicrometer-size particles in liquids [10-13]. As shown in recent studies [14-21], the liquid-phase environment of dispersed nanoparticles of wide bandgap semiconductors or insulators is very effective for a number of nonlinear optical effects. But the physical mechanisms involved, particularly with nonlinear optical processes in such media, are not entirely clear and require further study.

When placed in an electromagnetic field, particles in a microheterogeneous medium, with components that have different refractive indices, are subject to electrostrictive forces that could lead to the appearance of concentration flows [21-23]. Depending on the sign of the polarizability, these microparticles can be pulled in or pushed out of high electric field areas. At the same time that this electrostriction flow phenomenon occurs, thermal diffusion due to the temperature gradient [23] also occurs, but we do not consider thermal effects here. The concentration dynamics of colloidal particles in a periodic light field have been theoretically investigated in $[24,25]$, and similar problems within the small-perturbation approximation of the concentration were studied in $[26,27]$. In this paper, the restrictions of this approximation are removed.

Here, using the exact solution of the transformed diffusion equation for particles in the field of a light wave, we explore the dynamics of the particle concentration. The results allow us to find an explicit expression for the nonlinear part of the refractive index and calculate the focal length of the lens concentration formed by the self-interaction effects of the light field.

\section{Diffusion Equation}

The geometry of the problem considered in the paper is as follows. Electromagnetic radiation with some profile of the intensity falls on the cylindrical cell containing the investigated medium (Figure 1).

The equation describing the spatial and temporal variations of the nanoparticle mass concentration $C(r)$ can be written as a diffusion equation in terms of the external field 


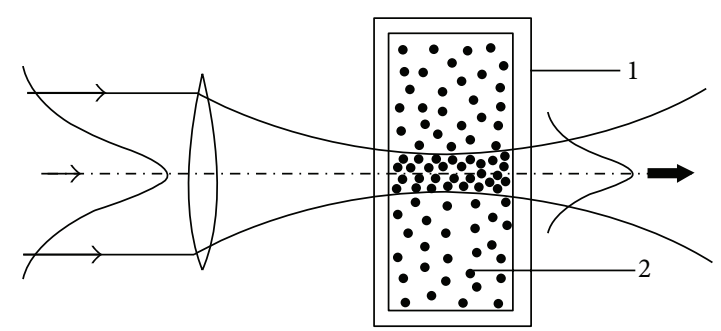

FIGURE 1: The geometry of the problem. 1: cell; 2: liquid medium with nanoparticles.

gradient (electrostrictive) forces as follows (we consider the axisymmetric case) [24]:

$$
\frac{\partial C}{\partial t}=D\left(\frac{\partial^{2} C}{\partial r^{2}}+\frac{1}{r} \frac{\partial C}{\partial r}\right)-\operatorname{div}\left(\gamma C \frac{\partial I}{\partial r}\right)
$$

where $C(r, t)=m_{0} / m, m_{0}$ is the total nanoparticle mass, $m$ is the mass of the whole fluid sample, $D$ is the diffusion coefficient, $\gamma=4 \pi \beta D / \bar{c} n k_{B} T, \beta$ is the polarizability of the particles, $k_{B}$ is the Boltzmann constant, $T$ is the temperature of the medium, $\bar{c}$ is the speed of light in vacuum, $n$ is the effective refractive index of the medium, $I=\widetilde{I}_{0} \exp \left(-r^{2} / r_{0}^{2}\right)$ is the intensity of the light beam, and $r_{0}$ is the beam radius. Equation (1) is sometimes referred to as the Smoluchowski equation [28].

To obtain the exact solution, consider the axial profile of the intensity of the beam in the paraxial Gaussian approximation $I=\widetilde{I}_{0}\left(1-r^{2} / r_{o}^{2}\right)$. Then, as it is small, we drop the term proportional to $\nabla C \nabla I$ (comparing $\nabla I \nabla C \approx$ $\delta C \delta I / l_{0}^{2}$ and $C \nabla^{2} I \approx C\left(\delta I / l_{0}^{2}\right)$, where $\delta C$ and $\delta I$ are small increments of concentration and intensity, resp., and $l_{0}$ is a characteristic length. We find that $\delta C \quad<\quad C$, which confirms the validity of the approximation). This is equivalent to neglecting convection, and in some cases, the experiment can be carried out in such a way that the influence convection is minimal. Equation (1), after the relevant changes for the initial and boundary conditions, can then be written as

$$
\begin{gathered}
\frac{\partial C}{\partial t}=D\left(\frac{\partial^{2} C}{\partial r^{2}}+\frac{1}{r} \frac{\partial C}{\partial r}\right)+\varphi C \\
C(r, 0)=C_{0},\left.\quad \frac{\partial C}{\partial r}\right|_{r=0}=0, \quad C\left(r_{0}, t\right)=C_{0}
\end{gathered}
$$

where $\varphi=4 \widetilde{I}_{0} \gamma / r_{0}^{2}$ and $C_{0}$ is the initial particle concentration.

The second boundary condition expresses the fact that the function $C(r, t)$ is finite at the origin and its symmetry axis of the cell. It means that particle flux is constant at the origin. The third boundary condition is due to the fact that, in the paraxial approximation, the concentration perturbations almost do not reach to the boundary.
We seek a solution in the form

$$
C(r, t)=\widetilde{C}(r, t) \exp (\varphi t)
$$

which leads to

$$
\begin{gathered}
\frac{\partial \widetilde{C}}{\partial t}=D\left(\frac{\partial^{2} \widetilde{C}}{\partial r^{2}}+\frac{1}{r} \frac{\partial \widetilde{C}}{\partial r}\right), \\
\widetilde{C}(r, 0)=C_{0},\left.\quad \frac{\partial \widetilde{C}}{\partial r}\right|_{r=0}=0, \\
\widetilde{C}\left(r_{0}, t\right)=C_{0} \exp (-\varphi t) .
\end{gathered}
$$

By using the Green's function of this problem [29], taking into account (4), and carrying out the relevant integration, the exact solution can be written as

$$
\begin{aligned}
C(r, t)= & 2 C_{0} \sum_{n=1}^{\infty} \frac{J_{0}\left(\mu_{n} r / r_{0}\right)}{J_{1}\left(\mu_{n}\right) \mu_{n}} \exp \left(\varphi-D \mu_{n}^{2} / r_{0}^{2}\right) t \\
& +2 C_{0} \sum_{n=1}^{\infty} \frac{J_{0}\left(\mu_{n} r / r_{0}\right)}{J_{1}\left(\mu_{n}\right) \mu_{n}^{2}\left(1-\varphi r_{0}^{2} / D \mu_{n}^{2}\right)} \\
& \times\left[\left(1-\exp \left(\varphi-D \mu_{n}^{2} / r_{0}^{2}\right) t\right)\right],
\end{aligned}
$$

where $J_{0}(x)$ is the Bessel function of order zero and $\mu_{n}$ are the roots of the equation $J_{0}(\mu)=0$. From the resulting solutions, we can see that, in the case of a positive exponent, $C(r, t) \rightarrow$ $\infty$ if $t \rightarrow \infty$, which is physically absurd. A negative sign can be provided in two ways: (1) $\varphi<D \mu_{n}^{2} / r_{0}^{2}$ or (2) $\varphi<0$. In the first case, using the explicit form of $\varphi$, we obtain a limit on the light intensity $\widetilde{I}_{0}<\mu_{n}^{2} \bar{c} n k_{B} / 16 \pi \beta$ (we also give an estimate below). In the second case, we must proceed from the explicit form of the polarizability, which for a sphere of radius $a_{0}$ can be written as [30]

$$
\beta=\varepsilon_{p} \frac{\varepsilon_{p}-\varepsilon_{l}}{\varepsilon_{p}+2 \varepsilon_{l}} a_{0}^{3},
$$

where $\varepsilon_{p}$ and $\varepsilon_{l}$ are the dielectric constant of the particles and liquid, respectively. Thus, case (2) is realized when $\varepsilon_{p}<\varepsilon_{l}$. 
Consequently, the transition to stationary conditions $(t \rightarrow$ $\infty)$ in (6) is possible and can be represented by

$$
\begin{aligned}
C_{\text {st }}(r) & =2 C_{0} \sum_{n=1}^{\infty} \frac{J_{0}\left(\mu_{n} r / r_{0}\right)}{J_{1}\left(\mu_{n}\right)\left(1-\varphi r_{0}^{2} / D \mu_{n}^{2}\right)} \\
& =C_{0} \frac{J_{0}(r \sqrt{\varphi / D})}{J_{0}\left(r_{0} \sqrt{\varphi / D}\right)}, \quad \text { if } \varphi>0, \\
C_{\text {st }}(r) & =C_{0} \frac{I_{0}(r \sqrt{\varphi / D})}{I_{0}\left(r_{0} \sqrt{\varphi / D}\right)}, \quad \text { if } \varphi<0,
\end{aligned}
$$

where $I_{0}(x)$ is the modified Bessel function of order zero. A series summation has been used to obtain (8) and (9) [31].

Note that the vanishing of the denominator in the second term does not lead to divergence as the uncertainty can be easily removed by means of l'Hôpital's rule. With regard to the convergence of (6) (omitting the details of the analysis), it can be argued that convergence is ensured by the exponents and the condition $r / r_{0}<1$. Furthermore, for small density perturbations, corresponding expressions for (8) and (9) were found in [26].

\section{Concentration Optical Nonlinearity and Effects of Self-Action}

If we take into account the dependence of the refractive index on the concentration, we can write

$$
n(C) \approx n\left(C_{0}\right)+\frac{\partial n}{\partial C}\left(C(r, t)-C_{0}\right) .
$$

Here, $n\left(C_{0}\right)=n_{0}$ is the unperturbed (linear part) of the refractive index and $\partial n / \partial C$ is a constant of the medium. Thus, referring to the above results for the concentration, we can say that the value of $n(C)$ is a result of the nonstationary, nonlinear function of the radiation intensity, which is not observed in the medium with an instant response. This is characteristic of media with a nonlocal (slow) nonlinearity mechanism (in our case, electrostriction).

Based on the inequality $C_{\mathrm{st}}(r=0)<1$ and using (8) or (9), we can obtain a limit on the light intensity, but it is then necessary to solve the irrational inequality $C_{0}<$ $J_{0}\left(r_{0} \varphi / D\right)$. By using the asymptotic behavior of the Bessel functions for small arguments, we get $C_{0}<1-r_{0}^{2} \varphi^{2} / D^{2}$. The possibility of such representation implies the following condition: $r_{0}^{2} \varphi^{2} / D<1$. Therefore, we first estimate the argument of the Bessel function by assuming $\beta=0.1 a_{0}^{3}$, $a_{0}=2 \times 10^{-9} \mathrm{~m}, n=1.3$, and $T=300 \mathrm{~K}$, and we obtain $4 \sqrt{\pi \widetilde{I}_{0} \beta / \bar{c} n k_{B} T} \approx 5 \times 10^{-6} \sqrt{\widetilde{I}_{0}}$. That is, up to an intensity of $\widetilde{I}_{0} \approx 10^{10} \mathrm{~W} / \mathrm{m}^{2}$, the argument of the Bessel functions is less than one and a decomposition is possible. Carrying out this expansion for (8) and (9) and substituting the result into (10), we obtain

$$
\begin{aligned}
& n(C) \approx n\left(C_{0}\right)+0.4 \pi \frac{a_{0}^{3}}{\bar{c} n k_{B} T}\left(1-\frac{r^{2}}{r_{0}^{2}}\right) C_{0} \widetilde{I}_{0} \frac{\partial n}{\partial C}, \quad \text { if } \Phi>0, \\
& n(C) \approx n\left(C_{0}\right)-0.4 \pi \frac{a_{0}^{3}}{\bar{c} n k_{B} T}\left(1-\frac{r^{2}}{r_{0}^{2}}\right) C_{0} \widetilde{I}_{0} \frac{\partial n}{\partial C}, \quad \text { if } \Phi<0 .
\end{aligned}
$$

Thus, within the paraxial approximation, the sign of the nonlinear part of the refractive index depends on the sign of the nanoparticle polarizability, that is, the values of the variables $\varepsilon_{p}$ and $\varepsilon_{l}$. Physical processes are related to the sign of the polarizability clearly presented in [15].

Note that the limiting intensity can be obtained by solving a simple hydrodynamic equation describing the motion of a particle subject to two forces: the gradient force exerted by the electric field of the light waves $F_{\nabla}=4 \pi \beta \nabla I / \bar{c} n$ and the Stokes drag force $F_{s}=6 \pi \eta a_{0} v$, where $\eta$ is the viscosity of the fluid and $v$ is the velocity of the particles. Thus, by determining the maximum speed of the particles (we omit the details of the calculation) and restricting the Reynolds number to $\operatorname{Re} \ll 1$, we get $\widetilde{I}_{0} \ll 15\left(\sqrt[4]{e} \bar{c} n / a_{0}^{2} \rho\right) \eta^{2}$, where $\rho$ is the particle density. Setting $\eta=1 \times 10^{-3} \mathrm{~kg} / \mathrm{m} \cdot \mathrm{s}$ (water), we get the desired estimate of $\bar{I}_{0} \ll 10^{16} \mathrm{~W} / \mathrm{m}^{2}$, which is consistent with our results. Using (11), we estimate the nonlinear part of the refractive index $n_{2}$ in the case of $r=0$ as

$$
n_{2}=0.4 \pi \frac{a_{0}^{3}}{\bar{c} n k_{B} T} C_{0} .
$$

Taking $a_{0}=2.34 \times 10^{-7} \mathrm{~m}, C_{0}=10^{-3}$, and $\partial n / \partial C=10^{-2}$ gives $n_{2} \approx 2 \times 10^{-13} \mathrm{~m}^{2} / \mathrm{W}$. Note that, in [32], a value of $n_{2} \approx$ $3 \times 10^{-13} \mathrm{~m}^{2} / \mathrm{W}$ which is consistent with our calculations was obtained experimentally for an aqueous suspension of latex particles of the same radius.

Self-action, in particular, is the formation of a lens in a layer of the medium. For small particulate concentrations, the effective index of refraction of the medium is proportional to the particle concentration and characterized by the parameter $(\partial n / \partial C)$.

These results allow us to calculate the focal length of the lens concentration formed in a layer of thickness $d$ as follows:

$$
\frac{1}{F}=-d\left(\frac{\partial n}{\partial C}\right)\left(\frac{\partial^{2} C}{\partial r^{2}}\right), \quad \text { for } r=0 .
$$

Then, as above, except in (8) and (9), where the arguments of the Bessel functions were quite small, after expanding in powers of the ratio $\Phi / D$ and the use of (13), we obtain an explicit expression for the focal length of

$$
\frac{1}{F}= \pm \frac{\varphi}{2 D} d C_{0}\left(1-\frac{r_{0}^{2} \varphi}{D}\right)\left(\frac{\partial n}{\partial C}\right) .
$$

In this case, the positive sign corresponds to the case with $\Phi>0$, that is, $\varepsilon_{p}>\varepsilon_{l}$, and the negative sign to $\Phi<0$, that is, $\varepsilon_{p}<\varepsilon_{l}$. 
Let us rewrite (15) in a more convenient form for estimation as follows:

$$
F=\frac{r_{0}^{4} \bar{c} n k_{B} T}{0.2 a_{0}^{3} d P} C_{0}\left(\frac{\partial n}{\partial C}\right)
$$

Assuming here that $r_{0}=10^{-4} \mathrm{~m}, C_{0}=10^{-2}, n=1.3$, $d=10^{-2} \mathrm{~m}$, and $P=10^{-2} \mathrm{~W}$ (along with the other quantities above), we then get $F \approx 10^{-4} \mathrm{~m}$, and so the focus of the concentration lens will be inside the medium.

As is well known, there is a discrete spectrum of nonlinear modes in the propagating beam, each of which takes its critical power from the self-focused beam. For example, the lowest axially symmetric mode has a critical power of [33]

$$
P_{\text {cr }}=1.3 \times \frac{\lambda^{2}}{32 \pi^{2} n_{2}} .
$$

For $\lambda=5.3 \times 10^{-7} \mathrm{~m}$ and using the value of $n_{2}$ derived earlier, we obtain an estimate of $P_{\mathrm{cr}} \approx 3 \times 10^{-3} \mathrm{~W}$. Knowing the explicit form of $n_{2}$, it can be argued that the power $P_{\text {cr }}$ does not depend on the width of the light beam.

\section{Discussion}

The approach that we have used lets us find an explicit solution of the spectral problem in (2) and (3), which describes the spatial-time dynamics of nanoparticles in a liquid medium and confirms that there is a response. Some previous theoretical descriptions of the self-action of light beams have relied on equations that assume a slowly changing amplitude and a nonstationary heat equation (a diffusion equation is used here), but obtaining an analytical solution for such systems is extremely difficult; problems involving heat nonlinearity are usually resolved by numerical methods. The explicit expression for the nonlinear part of the index of refraction that we have calculated here is caused by the action of the gradient force of the electric field of the light wave, and we have shown that it depends nonlinearly on the intensity.

With the exception of $[11,34,35]$, we could not find any publications that have experimentally studied the contribution to the concentration nonlinearity directly associated with electrostriction. In these published studies, the simultaneous contributions of both thermal diffusion and electrostriction were investigated. In two experiments [34, 35 ], the radiation source had a power of less than $500 \mathrm{~mW}$. We believe that with a higher radiation intensity for particles and a liquid with low absorption, it will be possible to distinguish the electrostriction contribution of the flow because the amount of light energy that is converted to heat is minimized. In our opinion, it is interesting that the intensity limitation appears naturally both in the thermodynamics and hydrodynamics. Note that this kind of limitation is absent in the self-action due to the heat nonlinearity of the medium, although both optical nonlinearity (heat and concentration) mechanisms are inertial.

In a previous paper [27], the dynamics of the nanoparticle concentration and the effects of self-action due to both thermal diffusion and electrostriction were described but within a small perturbation approximation. Therefore, for a more detailed study of these processes, it is necessary to solve (2) using (3) with the term that describes the thermal diffusion flux and to assess the contributions of the respective flows. This will be the topic of our future research.

\section{Conclusion}

We have described the spatial-temporal dynamics of nanoparticles in liquid-phase media and the electrostriction response using an approach that provides an exact solution to the spectral problem. Based on these results, we calculated an explicit expression for the nonlinear part of the refractive index due to the action of the gradient force exerted by the electric field of the light wave and an expression for the concentration lens focal length. We showed that this part of the index of refraction depends nonlinearly on the radiation intensity. In our view, the constraints on the value of the radiation intensity are inherent to the framework of thermodynamics and hydrodynamics.

\section{Acknowledgments}

The reported study was partially supported by the Ministry of Education and Science of the Russian Federation (Federal Target Program "Human Capital for Science and Education in Innovative Russia" for 2009-2013, State Contract no. 16.740.11.0317 and no. 16.740.11.0396). The authors are grateful for discussion with Professor Valeriy I. Ivanov and would like to thank Editage for providing editorial assistance.

\section{References}

[1] I. Krasnikov, A. Popov, A. Seteikin, and R. Myllylä, "Influence of titanium dioxide nanoparticles on skin surface temperature at sunlight irradiation," Biomedical Optics Express, vol. 2, no. 12, pp. 3278-3283, 2011.

[2] B. Apter, O. Guilatt, and U. Efron, "Ring-type plasmon resonance in metallic nanoshells," Applied Optics, vol. 50, no. 28, pp. 5457-5464, 2011.

[3] V. Krishtop, I. Doronin, and K. Okishev, "Improvement of photon correlation spectroscopy method for measuring nanoparticle size by using attenuated total reflectance," Optics Express, vol. 20, no. 23, pp. 25693-25699, 2012.

[4] J. H. Park, C. Park, H. Yu, Y. H. Cho, and Y. K. Park, "Dynamic active wave plate using random nanoparticles," Optics Express, vol. 20, no. 15, pp. 17010-17016, 2012.

[5] M. I. Shilomis, "Ferrofluids," Soviet Physics Uspekhi, vol. 17, no. 2, pp. 153-168, 1974.

[6] R. E. Rosensweig, Ferrohydrodynamics, Cambridge University Press, Cambridge, UK, 1985.

[7] J. C. Bacri, A. Cebers, A. Bourdon, G. Demouchy, B. M. Heegaard, and R. Perzynski, "Forced rayleigh experiment in a magnetic fluid," Physical Review Letters, vol. 74, no. 25, pp. 50325035, 1995.

[8] M. Kreuzer, T. Tschudi, W. H. de Jeu, and R. Eidenschink, "New liquid crystal display with bistability and selective erasure using scattering in filled nematics," Applied Physics Letters, vol. 62, no. 15, pp. 1712-1714, 1993. 
[9] R. S. Akopyan, N. V. Tabiryan, and T. Tschudi, "Optically induced hydrodynamic reorientation of liquid crystals and its applications for infrared detection and information storage," Physical Review E, vol. 49, no. 4, pp. 3143-3149, 1994.

[10] A. J. Palmer, "Nonlinear optics in aerosols," Optics Letters, vol. 5, no. 2, pp. 54-55, 1980.

[11] L. R. M. Vicari, "Dynamics of optical nonlinearity in water-inoil microemulsion," Japanese Journal of Applied Physics 1, vol. 40, no. 2, pp. 662-665, 2001.

[12] E. Freysz, M. Afifi, A. Ducasse, B. Pouligny, and J. R. Lalanne, "Giant optical non-linearities of critical micro-emulsions," Journal de Physique Lettres, vol. 46, pp. 181-187, 1985.

[13] L. Vicari, "Optical nonlinearity in a film of water in oil microemulsion," Optical Materials, vol. 18, no. 1, pp. 155-157, 2001.

[14] O. P. Mikheeva and A. I. Sidorov, "Optical nonlinearity of wide-bandgap semiconductor and insulator nanoparticles in the visible and near-infrared regions of the spectrum," Technical Physics, vol. 49, no. 6, pp. 739-744, 2004.

[15] R. El-Ganainy, D. N. Christodoulides, C. Rotschild, and M. Segev, "Soliton dynamics and self-induced transparency in nonlinear nanosuspensions," Optics Express, vol. 15, no. 16, pp. 10207-10218, 2007.

[16] Y. N. Kul'chin, A. V. Shcherbakov, V. P. Dzyuba, S. S. Voznesenskiy, and G. T. Mikaelyan, "Nonlinear-optical properties of heterogeneous liquid nanophase composites based on highenergy-gap $\mathrm{Al}_{2} \mathrm{O}_{3}$ nanoparticles," Quantum Electronics, vol. 38, no. 2, pp. 154-158, 2008.

[17] M. Matuszewski, W. Krolikovski, and Y. Kivshar, "Soliton interactions and transformations in colloidal media," Physical Review A, vol. 79, no. 2, Article ID 023814, 6 pages, 2009.

[18] M. Matuszewski, W. Krolikowski, and Y. S. Kivshar, "Spatial solitons and light-induced instabilities in colloidal media," Optics Express, vol. 16, no. 2, pp. 1371-1376, 2008.

[19] R. El-Ganainy, D. N. Christodoulides, Z. H. Musslimani, C. Rotschild, and M. Segev, "Optical beam instabilities in nonlinear nanosuspensions," Optics Letters, vol. 32, no. 21, pp. 31853187, 2007.

[20] C. Conti, G. Ruocco, and S. Trillo, "Optical spatial solitons in soft matter," Physical Review Letters, vol. 95, no. 18, Article ID 183902, 4 pages, 2005.

[21] V. I. Ivanov, Thermally Induced Mechanisms Recording Dynamic Holograms, Dalnauka, Vladivostok, Russia, 2006.

[22] A. Ashkin, Optical Trapping and Manipulation of Neutral Particles Using Lasers, World Scientific, Singapore, 2006.

[23] R. T. Schermer, C. C. Olson, J. P. Coleman, and F. Bucholtz, "Laser-induced thermophoresis of individual particles in a viscous liquid," Optics Express, vol. 19, no. 11, pp. 10571-10586, 2011.

[24] N. V. Tabiryan and W. Luo, "Soret feedback in thermal diffusion of suspensions," Physical Review E, vol. 57, no. 4, pp. 4431-4440, 1998.

[25] R. McGraw and D. Rogovin, "Response of an artificial Kerr medium to moving electromagnetic gratings," Physical Review A, vol. 35, no. 3, pp. 1181-1191, 1987.

[26] V. I. Ivanov and A. I. Livashvili, "Electrostriction mechanism of self-radiation in a liquid with nanoparticles," Bulletin of the Novosibirsk State University Series: Physics, vol. 4, no. 2, pp. 5860, 2009.

[27] V. I. Ivanov and A. I. Livashvili, "Self-Action of a Gaussian radiation beam in a layer of a liquid-phase microheterogeneous medium," Atmospheric and Oceanic Optics, vol. 23, no. 1, pp. 7-8, 2010.

[28] F. Rezakhanlou, "Pointwise bounds for the solutions of the Smoluchowski equation with diffusion," http://math.berkeley .edu/ rezakhan/44smolumoment3.pdf.

[29] A. D. Polyanin, Handbook of Linear Partial Differential Equations for Engineers and Scientists, Chapman \& Hall/CRC Press, Boca Raton, Fla, USA, 2002.

[30] L. D. Landau and E. M. Lifshitz, Fluid Mechanics, Elsevier Science, Oxford, UK, 1987.

[31] A. M. Prudnikov, A. Y. Brychkov, and O. I. Marychev, Integrals and Series: Special Functions, Nauka, Moscow, Russia, 1983.

[32] E. Freysz, W. Claeys, A. Ducasse, and B. Pouligny, "Dynamic grating induced by electrostrictive compression of critical microemulsions," IEEE Journal of Quantum Electronics, vol. 22, no. 8, pp. 1258-1262, 1986.

[33] N. I. Koroteev and I. L. Shumay, Physics of High-Power Radiation, Nauka, Moscow, Russia, 1991.

[34] L. R. M. Vicari, "Pump-probe detection of optical nonlinearity in water-in-oil microemulsion," Philosophical Magazine B, vol. 82, no. 4, pp. 447-452, 2002.

[35] L. R. M. Vicari, "Nonlinear optical characterization of cluster dynamic in water in oil microemulsion by a pump probe laser beam technique," European Physical Journal E, vol. 9, no. 4, pp. 335-340, 2002. 

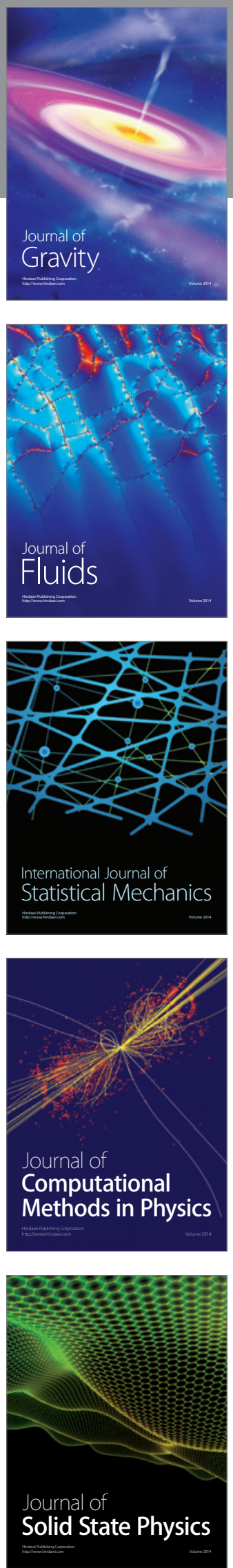

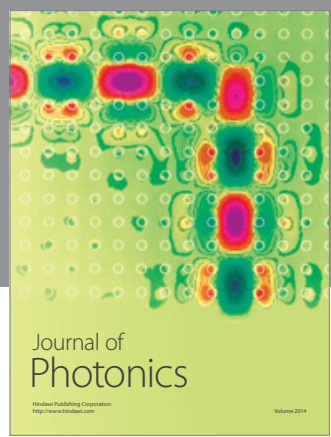

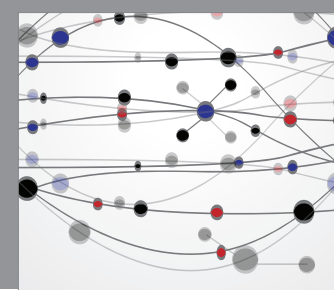

The Scientific World Journal

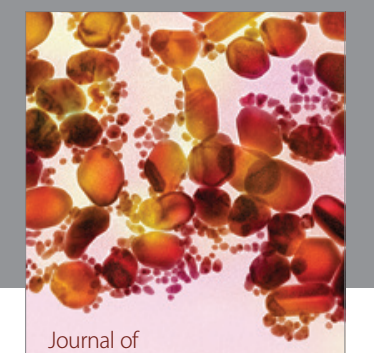

Soft Matter
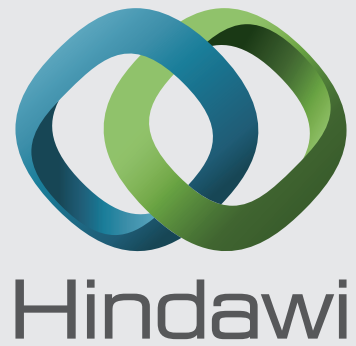

Submit your manuscripts at

http://www.hindawi.com
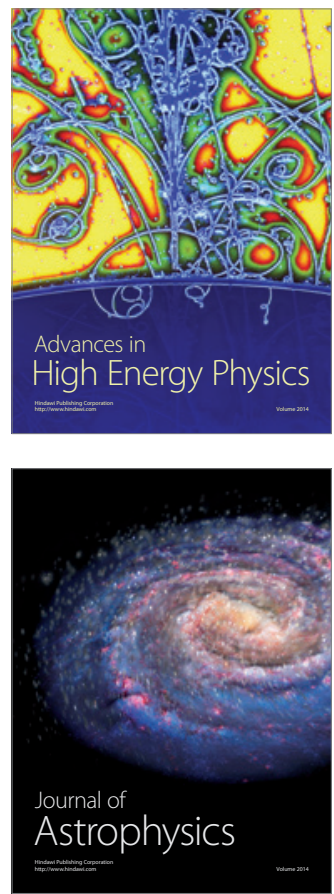
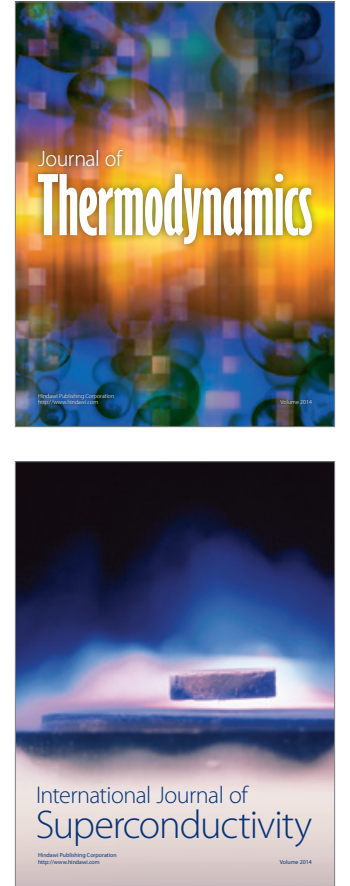
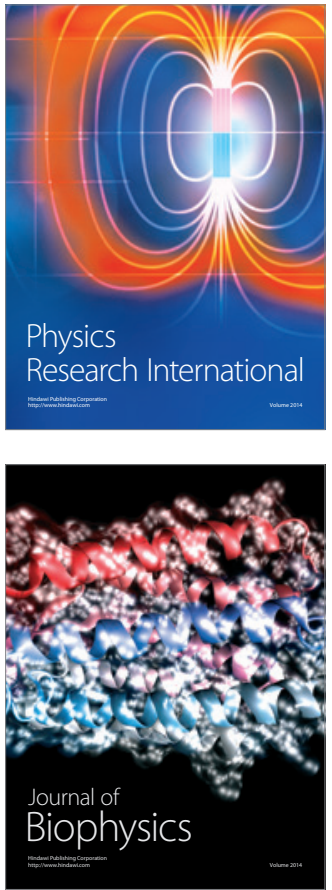
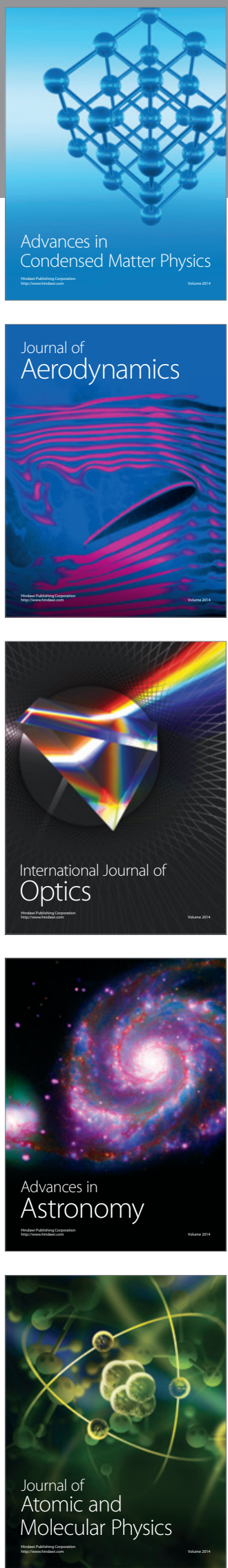